\title{
A Review of Bridge Management System: Deterioration Models
}

\author{
Gatesi Jean de Dieu ${ }^{1}$, Joao Agostinho Chingui ${ }^{2}$, Ergashev Botir ${ }^{3}$ \\ ${ }^{1}$ School of Transportation and Logistics Engineering, Southwest Jiatong University \\ Chengdu, Sichuan, P. R. China \\ Email: jados83 [AT] yahoo.fr \\ ${ }^{2}$ School of Transportation and Logistics Engineering, Chang'an University \\ People Republic of China \\ Email: familiachinguijo [AT] gmail.com \\ ${ }^{3}$ School of Transportation and Logistics Engineering, Southwest Jiatong University \\ Chengdu, Sichuan, P. R. China \\ Email: botiravto [AT] mail.ru
}

\begin{abstract}
In the last decades, in a large part of developed countries, a strong investment was made in the construction of infrastructures, namely bridges. However, the state of the road bridges changes with age due to several factors, which cause a progressive worsening of their degradation and a consequent decrease in the strength of the structure, if measures are not implemented that somehow cancel or counteract these effects, being of great importance correct management of the conservation status of these works of art.
\end{abstract}

Good managerial performance is essential especially in view of the limited financial resources or under the bias of their best use. Through computerized bridge management systems or "Bridge Management Systems", deterioration rates are introduced through algorithms that may vary according to the type of structure, its location, constituent material, or even the environment in which it is inserted, providing important subsidies for decision making. Deterioration Models that may be deterministic, such as least squares-based regression, or probabilistic, such as Markov chains are considered in this review paper. Other methodologies use artificial intelligence processes (natural algorithms or neural networks). The objective of the article is to describe the existing techniques, evaluating their limitations, specificities, and potential. Based on the awareness of the importance of deterioration models, it is possible to verify the fundamental role of routine inspections for the management of construction parks. The inspection of bridges can develop beyond the simple verification of the condition of the works in the present, providing subsidies for a better understanding of the behavior of these works over time. In the same way, inspection methods could provide for the collection of data relevant to the manager's knowledge containing information about possible decisive agents in the deterioration rates.

In the long term, the development and inclusion of explanatory variables in bridge management could bring great benefits to the industry. The development of mechanistic models used in conjunction with deterioration models could provide accurate results in predicting the state of condition. In addition, the development of these models would provide benefits in understanding the mechanisms of deterioration of the bridge components.

Keywords---- Bridge Management; Deterioration Models; Markov chains

\section{INTRODUCTION}

Bridges are structures that have the function of connecting points separated by natural or artificial obstacles. They are of great importance for the development of any country, as they help in the performance of roads, transporting people, food and others. Regardless of how bridges are built and the material used, the structures suffer degradation. They are affected by aging, fatigue, corrosion, incorrectly applied maintenance or not applying it due to increased loads, among other factors. Therefore, the management of these works is necessary to guarantee their safety and functionality through repair, restoration, rehabilitation and partial or integral replacement interventions, so that they can meet the minimum requirements for load capacity, safety, comfort and so on. The planning of corrective actions on bridges is of great importance in the transport sector due to the high costs and risks associated with these structures (MARKOW, et al., 2009) 
An appropriate way to minimize the richness of bridge interdiction is the establishment of a Bridge Management System, where it is necessary to carry out surveys or inspections to obtain data that indicate the status of the bridges; presenting the existing pathological problems, such as also possible solutions for the improvement of bridges, thus ensuring the appropriate development of their life cycle.

A Bridge Management System (BMS) is a software that seeks to reproduce and automate the procedures used by bridge managers. The figure 1 shows the main constituent elements and their main interrelationships in the bridge management process, linked to the use of BMS.

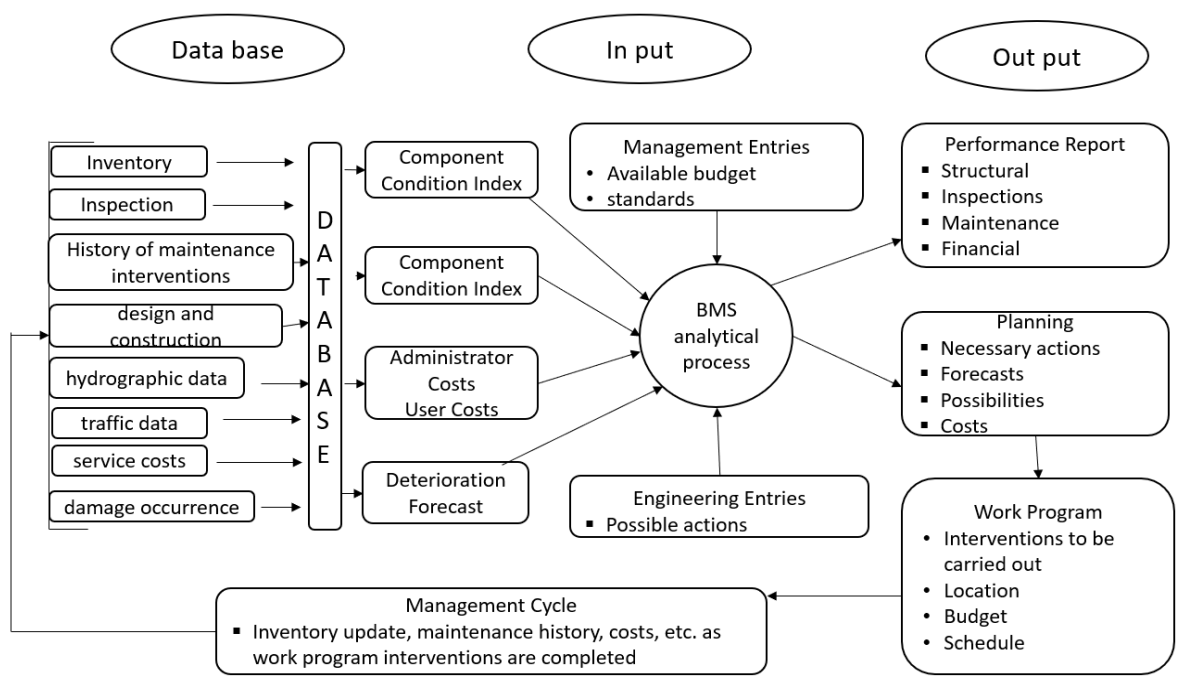

Figure 1 - Bridge management network, its elements and interrelations.

Source: Adapted from Austroads, 2013

The BMS is divided into three basic modules: the database, periodic inspections and evaluations and, finally, tools to support decision and management. The database is implemented in the system through the works inventory register, containing all available construction and design data (materials, construction system, capacity of load, dimensions, among others). In the inspections and evaluations module, periodic data related to the status of these works collected in inspections and possible changes in inventory data such as: change in traffic volume, changes in works, among others, are inserted. The first two modules are made up of data entry, with the main benefit of organizing information through its insertion according to a standardized format and in specific fields.

For management and support analysis in decision making, BMS use the cost of living analysis tool or Life-Cycle Costs Analysis (LCCA). LCCA's main approach is to consider not only the initial costs of buildings such as projects, construction and execution, but also long-term costs, such as operation, maintenance, repairs and rehabilitation (RYALL, 2001). The data resulting from the inspections are the input to the analysis models. The analysis models are divided into three parts, the cost module, the degradation module and the optimization module. The deterioration models and the analytical and numerical optimization tools make it possible to assess the performance of the bridges and measure risks, predict and plan interventions, determine priorities, calculate the costs involved in the works and plan budgets by optimizing the allocation of resources.

\section{THE BMS DETERIORATION MODULE}

After registering the works and their state of conservation to use the BMS optimization module, it is necessary to list the costs involved. Costs can be classified as direct, when referring to the specific maintenance, recovery and restoration services of these structures, or as indirect, when are related to the socioeconomic impacts arising from these interventions (ALMEIDA, 2013). The analytical process of each BMS may also present greater or lesser accuracy of the results depending on the algorithms used. However, the deterioration module has even more decisive importance in supporting management decisions, having a great impact on results, strongly dependent on its accuracy of its prediction (BU et al., 
2014). The deterioration model implemented in the BMS deterioration module is built using statistical tools based on data from periodic bridge inspections. Due to the characteristics of each country, such as climate, geographical position, deteriorating agents, construction techniques and materials, as well as the specific use of roads with certain types of fleet, speeds and loads, the processes and rates are particular of deterioration of these works in each region (YIANNI et al., 2016).This panorama presents the main difficulty encountered by managers worldwide in the use of deterioration models: it is essential to have a local database with the history of the change in the condition of these works over time. In addition to the database, for the preparation of the deterioration models there must be records of inspected works in sufficient quantity for an accurate determination of the deterioration rates of these structures. The absence of condition records for these works in the road sector may be due to the historical emphasis given to research in the paving area, with refinement of techniques, incorporation of new materials and research aimed at the deterioration of these structures and optimization of their conservation. The maintenance of special works of art (OAEs), especially bridges and overpasses on highways, are a relatively recent topic in several scientific researches, worldwide (ALMEIDA, 2013). A country with significant advances in this area of research, the United States of America only emphasized its work after an accident that occurred on the Silver Bridge bridge in 1967. Other countries also faced catastrophes due to the lack of maintenance of these works, especially accidents with the Seongsu bridge Bridge in 1994 (27 years later) in South Korea and the collapse of the Entre Rios bridge in Portugal in 2001, causing great national commotion. Because of the relatively recent interest, administrators have few records of bridge inspection. Besides, bridges usually observe insignificant changes in short intervals of inspections resulting in few changes in the conditions over time in the interval necessary for the elaboration of a predictive model of the rate of deterioration of the bridges in works parks (BUet al., 2014). As a consequence, despite the existing management resources in the BMS, the use of the software can be limited to the use of the input database and accessed by the managers to consult the records of the works and the data of the periodic inspections carried out. This restricted use is even indicated as typical in a report by the Organization of Australasian Road Transport and Transit Agencies (ASTROADS, 2015).

\subsection{Condition Index}

The existing bridge management programs are based on analyzes of the Condition Indexes or Technical Note (IC). The $\mathrm{CI}$ is a numerical index measured in an inspection of the performance of a given bridge. The CI can be assigned per element, per set of elements or for the entire work and reflects the final result of the assessment of the state of the work. Often, the $\mathrm{CI}$ adds a subjective attribution of the structural condition of the bridge, relating the value to the safety of the work being, therefore, the main data used for management decisions on the necessary intervention actions. The measurement of the structural state of the bridge can also be highlighted, using the IC scale to qualify the conditions of functionality and durability alone. In this way, more than one CI can be assigned to the same bridge.

The denominations of CIs and their scale vary according to the country. In the United States of America, the CI is called "Condition Rating - CR" and has 10 levels, starting from 0 (out of service) to 9 (excellent condition). In Brazil, the Condition Index is called "Technical Note" by the DNIT 010/2004-PRO and ABNT9452 / 2013 standards and can vary from 1 to 5, with 5 being assigned to structures in the best state and 1 being assigned to structures in the worst state. This numerical value is usually directly related to the safety of the work and, therefore, it becomes the main data used for management decisions about the necessary intervention actions.

\subsection{Types of Deterioration Models}

There are several methods for determining bridge deterioration models, that can be empirical or mechanistic. Empirical models use experimental data, while mechanistic models are developed from theoretical models associated with degradation mechanisms.

These methods can be classified into deterministic, stochastic and artificial intelligence deterioration models (SETUNGE, et al., 2011). Deterministic deterioration models are obtained through statistical and regression models, which seek to describe trends based on historical data on structural deterioration. The functions resulting from these models consider that the work starts with a value of $100 \%$ and ends its useful life with a value of zero.

- In the stochastic deterioration models, the condition states are understood as random input variables, attributing probabilities of the occurrence of a certain state in the sample universe.

- The deterioration models using artificial intelligence seek to find relationships between the Condition Index and internal and external variables to the bridges. The internal variables to the bridges are those resulting from the structure and its characteristics (span size, age of the bridge, construction system, among others).

- For the development of deterministic models, linear and, non-linear regressions, linear extrapolations and curve adjustments can be performed. 


\section{a) Deterministic deterioration models}

Deterministic deterioration models are obtained through statistical and regression models that seek to describe trends based on historical data on structural deterioration. The functions resulting from these models consider that the work starts with a value of $100 \%$ and ends its useful life with a value of zero. The main stochastic method used in deterministic models is the third order polynomial regression:

$I C_{i}=\beta_{0}+\beta_{1} t_{j}+\beta_{2} t_{j}^{2}+\beta_{3} t_{j}^{3}(1)$

IC being the note of the bridge $i=1, \ldots, 34$, and the time $j=1, \ldots, 7$.

Polynomial regression considers the history of each bridge based on this knowledge for the projection of the group's behavior. This possibility of dealing results in a more accurate description of the individual's deteriorating process. Another advantage of the method is the possibility of checking for possible correlations between the deterioration rates obtained with the internal or external factors of the structures. For example, the rate variation can be verified according to different types of structural systems, materials, age of the works, traffic characteristics, environmental aggressiveness and other possible influencing agents. For the use of regression as an inferential form, some assumptions must be observed such as independence between observations, normality and constant variance. As the data has a longitudinal structure, an inferential model must take into account the existing dependency structure on the same bridge.

\section{b) Probabilistic deterioration models using Markov Processes or stochastic deterioration models}

In the process of structural deterioration, several agents can hardly have their influence rates individually studied. In this sense, the phenomenon of deterioration of bridges has a probabilistic bias, which is the main justification for the greater use of probabilistic models in research in this area of science. The main stochastic models are divided into Markov Models, Semi-Markov Models, Weibull Survival Models, Markov-Weibull Hybrid Models, and Gamma Process Deterioration Models (ASTROADS, 2015). Among the probabilistic models and the decision processes, the Markov processes stand out in the identification of optimal structural projects and their associated maintenance policies. This approach is widely used, as it can identify the trend according to a probabilistic approach in addition to resulting in a computationally attractive mathematical model (TAO et al, 1994).

Markov processes are stochastic processes, that is, they are processes that characterize the behavior of a system over time with defined probabilistic considerations (ALMEIDA, 2013). In the time variable, Markov processes can be characterized as discrete or continuous according to the study variables. For the time variable, its nature can be discrete, with $X(t), t=$ $0,1,2, \ldots$ or continuous, with $X(t), t>0$. Input variables can also be continuous or discrete. Although the deterioration process has a continuous character, the use of discrete CIs is widely used to measure the condition of the structure, reducing the complexity of continuous monitoring of this process (MADANAT, et al., 1995).

When the process considers independence over time, that is, there is no change in its result when promoting a displacement in time, this is called a stationary process. There are two types of deterioration models using discrete state Markov processes: time-based models and state-based models. State-based models are deterioration models that use the chain Markov process, and study the probability that a given bridge will to maintain its CI or change it within a fixed time interval $\Delta t$. Markov chains are also suitable and widely used to develop statistical models of deterioration of various types of infrastructure materials, such as pavements, bridges, buildings, among others. Time-based models are deterioration models that predict the probability of the time interval that a bridge takes to make a state transition or condition index (ALMEIDA, 2013).

The probability of an element moving from state $i$ at an initial time to a state $\mathrm{j}$ at a time $\Delta \mathrm{t}$ is given by:

$P_{(\Delta t) i j}=P_{r}\left(X_{\Delta t}=j \mid X_{\Delta t}=i\right)(2)$

and similarly, the probability of an element remaining in state $\mathrm{i}$ at a time $\Delta \mathrm{t}$ is given by:

$$
\mathrm{P}_{(\Delta \mathrm{t}) \mathrm{ii}}=\mathrm{P}_{\mathrm{r}}\left(\mathrm{X}_{\Delta \mathrm{t}}=\mathrm{i} \mid \mathrm{X}_{\Delta \mathrm{t}}=\mathrm{i}\right)(3)
$$

Each element $p_{i j}$ represents the probability that the bridge IC will pass from $i$ to $j$ during a determined time interval $(\Delta t)$. The structure of the MPT (Matrix of Probability Transition) is described by the equation matrix: 
$P=\left[p_{i j}\right]=\left[\begin{array}{cccc}p_{11} & p_{12} & \cdots & p_{1 n} \\ p_{21} & p_{22} & \cdots & p_{2 n} \\ \vdots & \vdots & & \vdots \\ p_{m 1} & p_{m 2} & \cdots & p_{m n}\end{array}\right]$ (4)

The diagram in Figure 2illustrates the probabilities of a transition matrix for four levels with a numerical example for better understanding, considering $\mathrm{CI}=4$ as the worst condition and $\mathrm{CI}=1$ as the best condition.

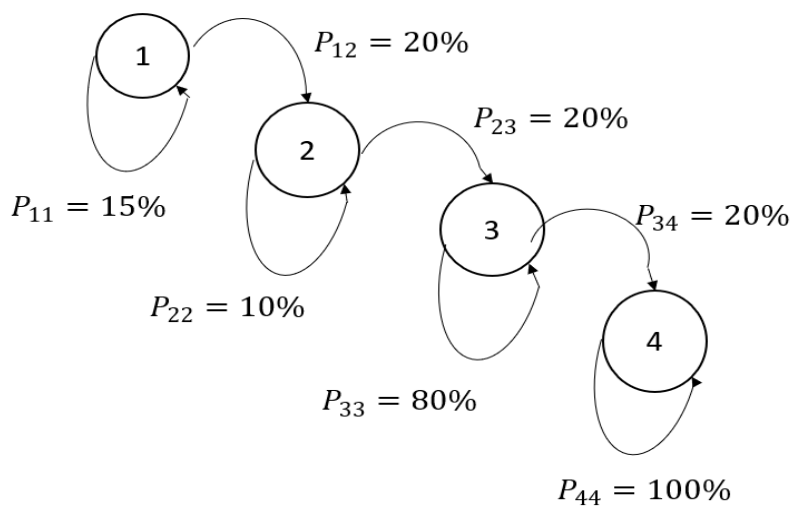

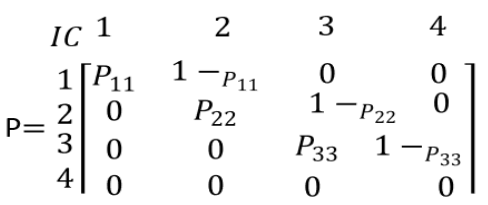

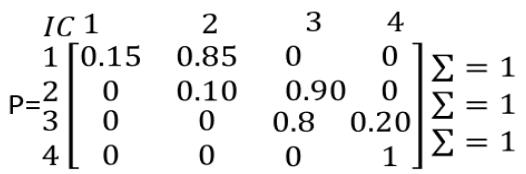

Figure 2- Structure of a Probability Transition Matrix.

There are some properties of MPT for application in bridge deterioration models, highlighted in the study by (MADANAT, et al., 1995):

- $\quad 0 \leq P_{i j} \leq 1$ with $i=1,2, \ldots, k$ where $k$ is the total number of levels $\operatorname{IC}(5)$

- $\quad \sum_{i}^{k} p_{i j}=1$ with $i=1,2, \ldots, k(6)$

- $\Delta t=t_{f}-t_{i n}(7)$

The Equation 5limits that the MPT for the elaboration of a deterioration model must be a square matrix with the number of columns (or rows) equal and equivalent to the possible CIs. The matrix model must be upper triangular with the elements below the main diagonal equal to zero in the specific case of the best state being IC $=1$ and worst $\mathrm{IC}=4$. For contrary $\mathrm{CI}$ assignments, as in the Brazilian case, the matrix must be upper triangular. The MPT is also a non-negative matrix because they are probabilities and has non-zero elements on its main diagonal. Finally, the probability for the last element of the matrix, located in the region on the lower right should be $100 \%$.

\section{c) Artificial Intelligence deterioration models}

Artificial intelligence models use computation techniques in an attempt to find similar patterns between data, relating the CI with variables called "external" to bridges, but which are known to be associated with the agents of their degradation. The methods used in this model seek to reduce the uncertainties resulting from CI databases with insufficient history for acceptable reliability of the deterioration model.

The main artificial intelligence models are those used by neural networks (Artificial Neural Network - ANN) associated with the Past IC Prediction Model (Backward Prediction Model - BPM) and the so-called Historical Based Models (Casebased Reasoning Models - CBR) (MORCOUS, et al., 2002).

Some research seeks to increase the accuracy of the models for small CI databases. The BPM method, for example, basically consists of creating a historical database from existing data, created according to relationships found by neural network algorithms, thus forming a database with a greater number of variables and greater reliability (BU, et al., 20014). The generated historical data are then submitted to the same algorithm and the dispersion of the model itself is verified. Figure 3 illustrates this mechanism. 
Year of

Construction
Current data

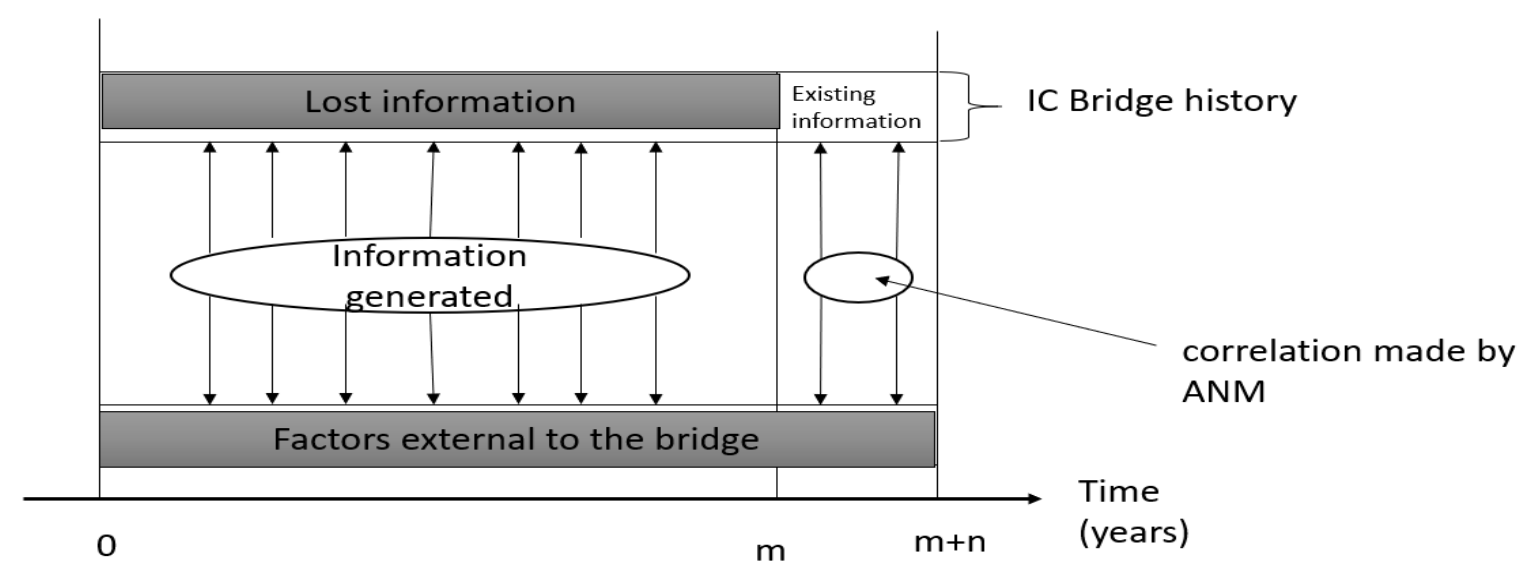

Figure 3 - BPM mechanism.

Source: Adapted from: Bu et al. (2013)

\section{CONCLUSION}

The perceived and real barriers to achieving the effective implementation ofdeterioration need to be identified and weighed against the value that can be added to asset management decision making. One of the mainbarriers to introducing any of these systems is the availability of data. To take advantage of the potential benefits of deterioration models, it would be necessary to a significant effort to capture bridge condition data, consistently, to improve its availability and uniformity. The existence of explanatory variables and standardized records can guarantee a valuable and significant set of data, important for the development of consistent deterioration models. Based on the awareness of the importance of deterioration models, it is possible to verify the fundamental role of routine inspections for the management of construction parks. The inspection of bridges can develop beyond the simple verification of the condition of the works in the present, providing subsidies for a better understanding of the behavior of these works over time. In the same way, inspection methods could provide for the collection of data relevant to the manager's knowledge containing information about possible decisive agents in the deterioration rates.

Often relatively easy to record data can have a strong impact on management decisions, resulting in savings and better management of resources in various situations. On the other hand, resources can be wasted if there is no concern to collect certain information in the field. In the long term, the development and inclusion of explanatory variables in bridge management could bring great benefits to the industry. The development of mechanistic models used in conjunction with deterioration models could provide accurate results in predicting the state of condition. In addition, the development of these models would provide benefits in understanding the mechanisms of deterioration of the bridge components.

\section{ACKNOWLEDGEMENT}

This research paper has been prepared by referencing the different previous research studies as review. The many thanks are expressed to all past researchers and practitioners for their hard work on this field. Our thanks also are addressed to the authors of this paper who worked together to complete this research work. Finally, we acknowledge one to another for their support in achieving this research review paper. 


\section{REFERENCES}

[1] Bridge Management System [Report] / auth. TRANSPORTATION RESEARCH BOARD. - Washington,D.C : National Cooperative Highway Research Program, December 1987.

[2] Application of Artificial Intelligence for Bridge Deterioration Model [Journal] / auth. Chen Zhang [et al.] // The Scientific World Journal. - 2015.

[3] Artificial Intelligence Models and Bridge [Report] / auth. WINM EMILY K and BURGUENO RIGOBERTO. [s.l.] : Department of Civil and Environmental Engineering, 2013.

[4] Bridge Deterioration Prediction Using Markov-Chain Model Based on the Actual Repar Status in Shanghai [Conference] / auth. LI L [et al.] // Annual meeting of the transportation Research Board. - Shangai, China : [s.n.], 2016.

[5] Bridge management system based on cycle cost [] / . - Porto, PortugalUniversity of Porto, School of Engineering (FEUP), 2013

[6] Bridge Management Systems for Transportation Agency Decision [Report] / auth. MARKOW M. J and HYMAN W. A. - Washington, D. C : National Cooperative Highway Research Program, 2009.

[7] Bridge Management Using Performance Models [Book] / auth. ARRB Group. - Sydney : Austroads Ltd, 2013.

[8] Bridge Management. Elsevier, [Book] / auth. RYALL M. J. - 2001. - ISBN: 075065077.

[9] Concrete Bridge Deterioration Prediciton using Markov Chain [Journal] / auth. SETUNGE S and HASAN M S // RMIT University. - 2011.

[10] Concrete Bridge Deterioration Prediction using Markov Chain Approach. [Report] / auth. SETUNGE SUJEEVA and HASAN MD SAEED. - Melbourne, Austrália : RMIT University, 2011.

[11] Condition Improvement Measurement Using the Condition Evaluation Criteria of Concrete Bridge Decks [Journal] / auth. YOON Y and HASTAK M // Journal of Transportation Engineering. - [s.1.] : Journal of Transportation Engineering,, 2016. - pp. P.142-153.

[12] Development of an Integrated Method for Probabilistic Bridge-Deterioration Modeling. [Journal] / auth. BU G [et al.] // Journal of Performance of Constructed Facilities. - [s.l.] : Journal of Performance of Constructed Facilities, 20014. - pp. p.330-340.

[13] Development Of Deterioration Models For Bridge Decks Using [Report] / auth. GHODOOSIPOOR F. - Quebec, Canada : Concordia Univesity, 2013.

[14] Estimation of Infrastructure Transition Probabilities from Condition Ranting Data. Journal of Infrastructure Systems, [Report] / auth. MADANAT S [et al.]. - 1995.

[15] Handbook of Concrete Bridge Management [Report] / auth. BRANCO F, A and BRITO J. - [s.l.] : American, 2003.

[16] Improved bridge deterioration models, predictive tools and costs [Report] / auth. KATZE R, NGO H and SESKIS J. - [s.l.] : Australia, 2015.

[17] Improved Bridge Deterioration Models, Predictive Tools and Costs [Report] / auth. AUSTRALASIAN ROAD TRANSPORT AND TRAFFIC AGENCIES - ASTROADS. - Sydney,Australia : Research Report, 2015.

[18] Incorporating local environmental factors into railway bridge asset management [Journal] / auth. YIANNI PANAYIOTI C [et al.] // Engineering Structures. - [s.1.] : Elsevier, 2016. - pp. p.362-373.

[19] Life-cycle assessment of highway bridges [Journal] / auth. MORCOUS G, LOUNIS Z and MIRZA M. S // Institute for Research in construction IRC, National Research Council Canadá. - 2002. - p. p.18.

[20] Modeling Bridge Deterioration with Markov Chains [Report] / auth. Cesare Mark and Santanarina J Carlos. - [s.l.] : ResearchGate, 19 February 2015.

[21] Optimal maintenance strategies for bridge networks using the supply and demand approach [] / // ResearchGate 
[22] Pavement and Asset Management [Book] / auth. Crispino Maurizio. - Chennai,India : CRC Press/Balkena, 2019.

[23] Performance prediction of Bridge Deck Systems Using Markov Chains [Journal] / auth. MORCOUS G // Journal of Performance of Constructed Facilities. - 2006. - pp. p146-155.

[24] Pontis Bridge Management [Report] / auth. AASHTRO. - Washington, D.C : American Association of State Highway and Transportation Officials, , 2009.

[25] The Manual for Bidge Evaluation. Washington,USA [] / AMERICAN ASSOCIATION OF STATE HIGHWAY AND TRANSPORTATION OFFCIALS-AASHTO. - p.3-3, 3-6

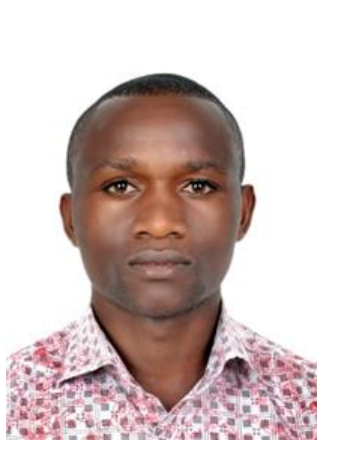

\section{AUTHORS BIOGRPAHY}

Environmental Technology (College of Science \& Technology-University of Rwanda) in 2010, Master's degree holder in Civil (Transportation Engineering), L.D College of Engineering -Gujarat Technological University, India in 2015. He worked as Assistant Lecturer at University of Rwanda. At present is Doctorate student with majoring in Traffic Engineering under the school of Transportation and Logistics, Southwest Jiatong University-P.R.China. He has published more than eight research papers in national and international journals. He has also presented the one more paper in international conference, India. Interested area of research: Traffic Engineering, Intelligent Transportation System, Road Traffic Accidents Analysis and Highway pavement materials.

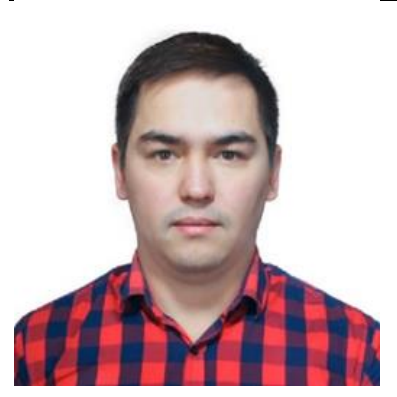

Mr. ERGASHEV BOTIR ZOKIR O'GLI, was born 1990 in Tashkent which is capital city of Uzbekistan, his Bsc in Transportation and Traffic Engineering (Tashkent Institute of Design, Construction and Maintenance of Automotive roads, Uzbekistan) in 2009, master's degree holder in Civil (Road construction), in the same university, Uzbekistan in 2015.At present is Doctorate student with majoring in Transportation Engineering under the school of Transportation and Logistics, Southwest Jiatong University-P.R.China. He has several research papers in national and international journals.

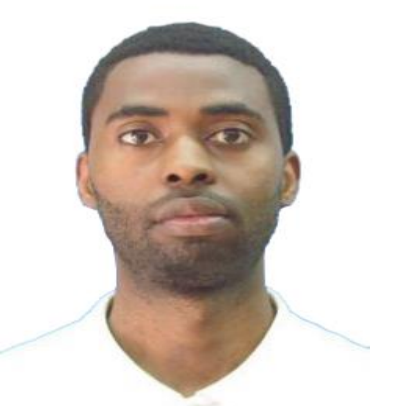

Mr. JOÃO AGOSTINHO CHINGUI, was born 1992 in LUBANGO one of the cities of Angola, his completed Bsc in Transportation and Traffic Engineering (School of Transportation and LogisticsSouthwest Jiaotong University-P.R.China) in 2019. At present is Muster student with majoring in Traffic Engineering under the School of Transport Engineering, Chang'an University-P.R.China. 